\title{
Vibratory dynamics of four types of excised larynx phonations
}

\section{Lin $\mathbf{L i}$}

College of Oceanography and Environmental Science, Xiamen University, Xiamen, Fujian 361005, P. R. China.

E-mail: lilin_xmu@163.com.

\section{Yu Zhang*}

Key Laboratory of Underwater Acoustic Communication and Marine Information Technology of the Ministry of Education, Xiamen University, Xiamen, 361005, P. R. China.

Also at State Key Laboratory of Marine Environmental Science, Xiamen University, Xiamen, 361005, P. R China.

*Corresponding author. E-mail: yuzhang@xmu.edu.cn. Tel : (+86) 15160098758

\section{William Calawerts}

Division of Otolaryngology-Head and Neck Surgery, Department of Surgery, University of Wisconsin School of Medicine and Public Health, Madison, Wisconsin, 53726, USA

E-mail:wcalawerts@wisc.edu

\section{Jack J. Jiang}

Division of Otolaryngology-Head and Neck Surgery, Department of Surgery, University of Wisconsin School of Medicine and Public Health, Madison, Wisconsin, 53726, USA.

E-mail: jjjiang@wisc.edu. 


\begin{abstract}
:
Objective: There are four types of signals that are typical representations of vocal fold vibratory patterns. Type 1 signals are nearly periodic, type 2 signals contain subharmonic properties, type 3 signals are chaotic, and type 4 signals are characterized as white noise. High-speed imaging allows detailed observation of these vocal fold vibratory patterns. Therefore, high-speed imaging can explore the vibratory mechanism behind each of the four types of signals.
\end{abstract}

Methods: The glottal area time series of the four types of vocal fold vibrations was calculated from high-speed images of ten excised canine larynges. Nonlinear dynamic parameters of correlation dimension $\left(\mathrm{D}_{2}\right)$ and Kolmogorov entropy $\left(\mathrm{K}_{2}\right)$ were used to quantify the characteristics of the glottal areas and acoustical signals for each voice signal type.

Results: The correlation dimension and Kolmogorov entropy of the glottal areas and acoustical signals for type 1,2, and 3 voice signals were consistent with the results of previous studies. Interestingly, there was a difference between the glottal area and acoustical signals of type 4 voice signals $(p<0.001)$. Both the correlation dimension and Kolmogorov entropy of the type 4 glottal area were close to 0 . In contrast, the type 4 acoustical signals had an infinite correlation dimension and a Kolmogorov entropy that was close to 1 .

Conclusions: Turbulence in the vocal tract creates high-frequency breathiness, causing noise in the acoustical signal of type 4 voice, proving that the acoustical signal does not represent the motion mechanism behind type 4 voice. The results of this study demonstrate that high-speed imaging can provide a more accurate representation of the type 4 vocal fold vibratory pattern, and a more effective method to explore the mechanism of type 4 signals.

Keywords: High-speed imaging; Type 4 signal; Correlation dimension; Kolmogorov entropy 


\section{Introduction}

In 1995, Titze introduced a three tiered classification scheme [1] to quantify voice signals. According to his scheme, type 1 signals were defined as nearly periodic, type 2 signals exhibited strong modulations or subharmonic that approached the fundamental frequency in energy, and type 3 signals were irregular or aperiodic. Recently, Sprecher [2] added a fourth voice type to Titze's voice classification scheme. Sprecher updated type 3 voice to be defined as a signal that is chaotic with a finite dimension, and type 4 voices were defined as chaotic and dominated by stochastic noise features with infinite dimension. In contrast to the type 3 voice signals that are characterized by band limited spectra with the energy centralized to lower frequencies, type 4 voice signals show a searing of energy across a broader range of frequencies, resembling that of broadband white noise.

Previous research has effectively analyzed the acoustic signals of the first three voice types using perturbation and nonlinear dynamic analysis methods [3-8]. It has been proven that nonlinear dynamic methods are useful in quantifying complex voice systems, such as type 3 and type 4 voice signals [3-8]. However, due to complicating factors such as turbulent noise and vocal tract filtering, the collected acoustic voice signals are not able to provide information about the vibratory dynamics of each signal type, leading to the development of other analysis methods. Since visualization of vocal fold vibrations is a powerful tool in the diagnosis of laryngeal pathology, digital kymography (DKG) [9-11] and high-speed imaging [12-15] have emerged as effective analysis methods in recent years. These imaging methods allow the objective evaluation of vocal fold vibratory parameters, leading to a better understanding of the mechanisms of disordered voice production and enhanced assessment of laryngeal pathology.

Sprecher [2] systematically analyzed the four types of voice signals using spectrum and acoustical analysis in 2010. Additionally, Zhang [16] provided a complement to this traditional signal classification techniques by using DKG for vibratory classification of voice signals.

The purpose of this study is to incorporate Sprecher's acoustical classification scheme into the vocal fold vibratory pattern classification method presented by Zhang and investigate the differences between the vibratory patterns and acoustical signals of type 4 voice signals. Vibratory images and corresponding acoustical signals of the four types of voice signals from ten excised canine larynges were recorded using high-speed imaging and a microphone. The glottal area time series of the four 
voice signal types were extracted from high-speed images and nonlinear dynamic analysis was performed to quantify the glottal area and acoustical signal time series.

\section{Methods}

\subsection{Excised Larynx tests}

The excised larynx experimental setup is shown in Fig. 1(a). Ten canine larynges were harvested from healthy laboratory dogs and used in an experimental trial 12 to 36 hours after excision. Using a hose clamp, a segment of each trachea was secured to a pipe. A conventional air compressor, conditioned to $25-30^{\circ} \mathrm{C}$, at $95 \%$ relative humidity and small than $45 \mathrm{~dB}$ noise level, was used to generate airflow. For each trial, subglottal pressure and flow were held constant until phonation was maintained for accurate high-speed measurement. The vocal fold vibratory images were recorded by a high-speed digital camera (Phantom MIRO M110, Ametek) at a sampling rate of 4000 frames/s with a resolution of $512 \times 256$ pixels. The videos were recorded at $30 \mathrm{~cm}$ distance from each larynx. The microphone was used to record the acoustical signals during each trial.

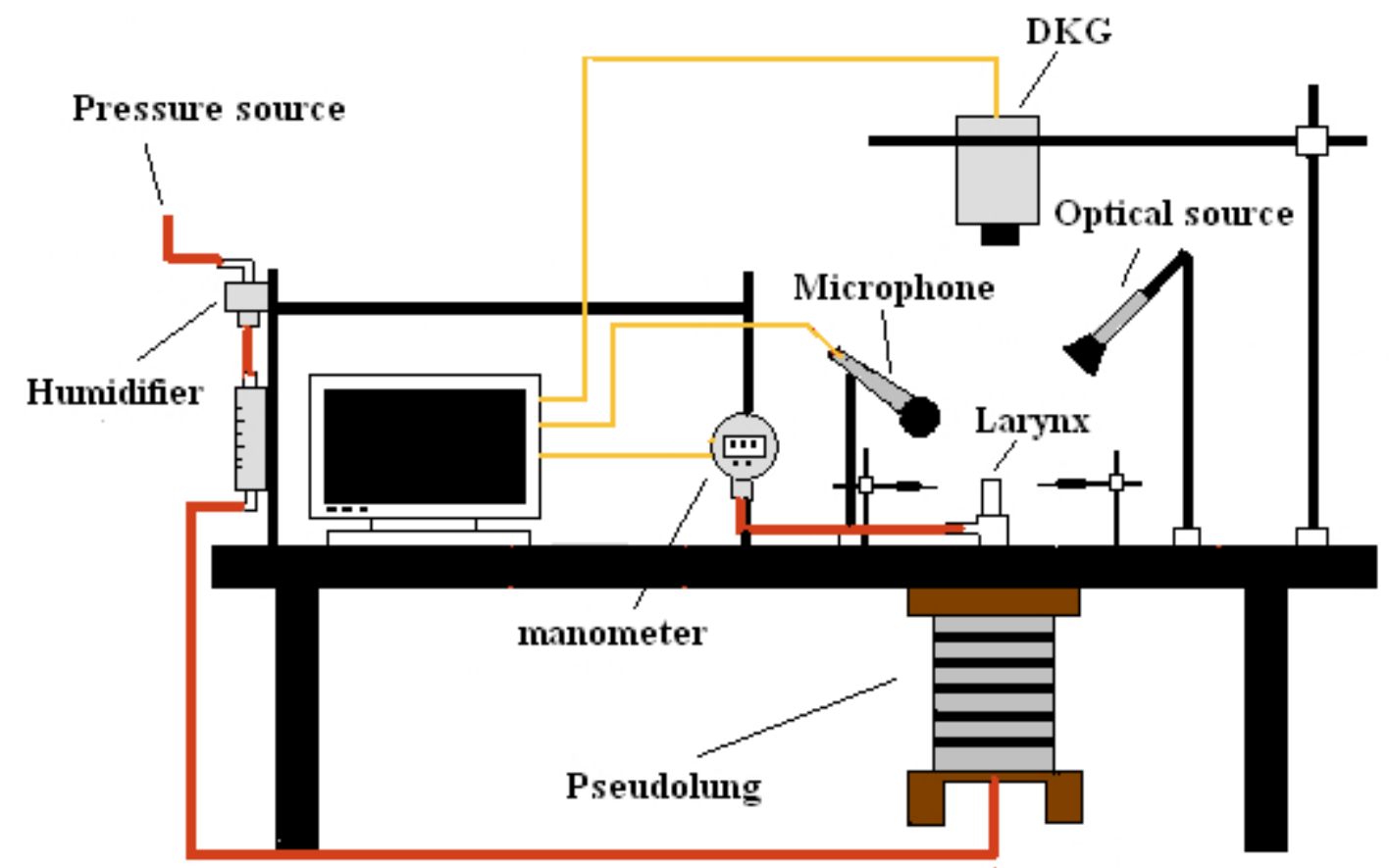

(a) 


\section{Type 1}

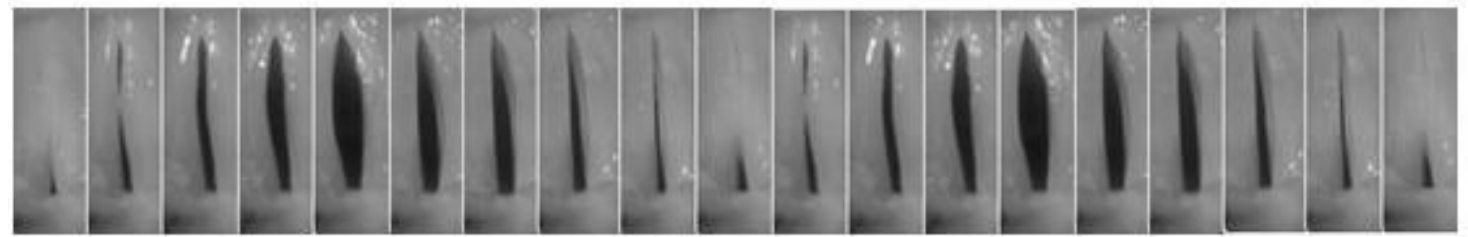

Type 2

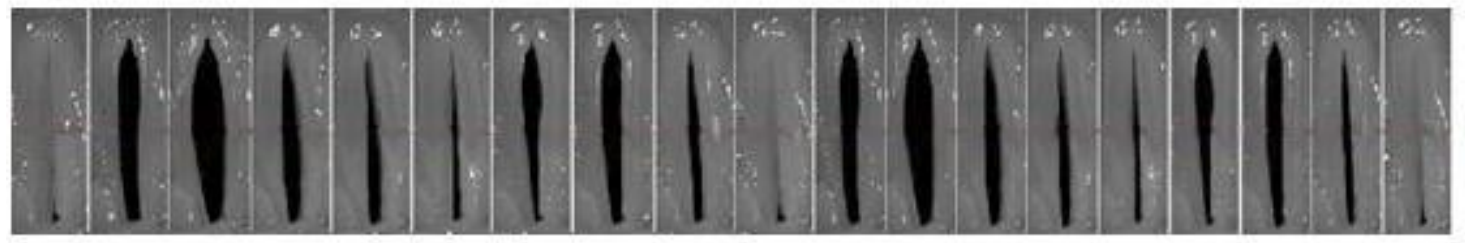

Type 3

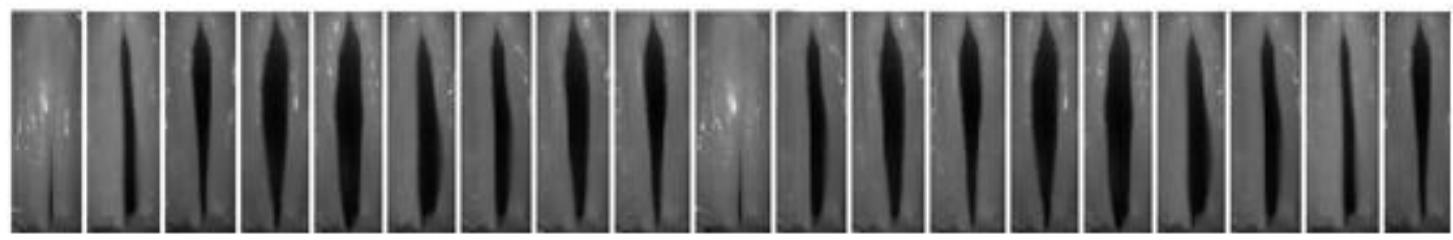

Type 4

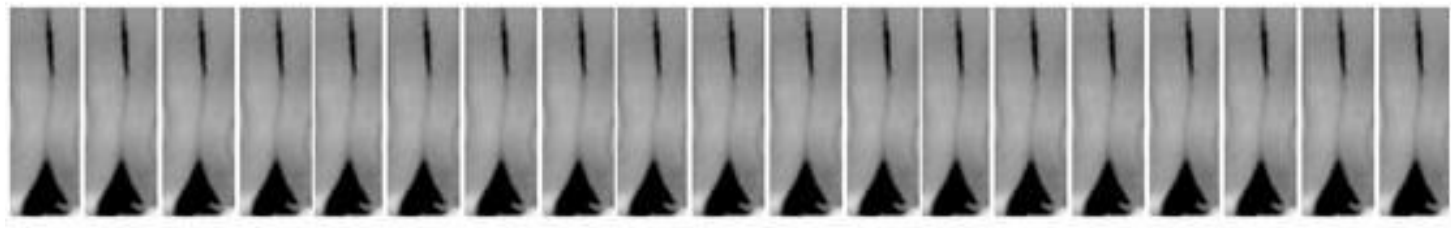

(b)

Fig.1 (a) An illustration of the excised larynx setup. (b) 19 frames of high-speed images of type 1, 2, 3 and 4 vibration of the vocal fold

In order to collect all four types of acoustical signals and high-speed images, the excised larynx experiments were conducted at subglottal pressures of $10 \mathrm{~cm} \mathrm{H}_{2} \mathrm{O}, 30 \mathrm{~cm} \mathrm{H}_{2} \mathrm{O}, 40 \mathrm{~cm} \mathrm{H}_{2} \mathrm{O}$ and $40 \mathrm{~cm}$ $\mathrm{H}_{2} \mathrm{O}$ with a $2 \mathrm{~cm}$ wide gap. During these experiments, all four types of acoustical signals and corresponding high-speed images were captured according to the classification scheme reported by Sprecher [2]. As demonstrated in Fig 1(b), type 4 signals exhibited little vibration. To research the vibratory patterns of all four voice signal types, the glottal edges from the high-speed images were extracted using Lagrange interpolation analysis with Canny image edge detection as previously reported [17]. The glottal areas were computed by counting the pixels within glottal edges of vocal fold. 


\subsection{Nonlinear dynamic analyses of glottal area and acoustical time series}

As mentioned, the purpose of this paper was to find a quantitative parameter capable of reflecting the difference between the vibratory pattern and the acoustical signal of all four types of voice signals. As previous research has reported, nonlinear dynamic analyses are usually used to describe the dynamic characteristics of a vocal fold system. In this paper, the numerical algorithms of phase space reconstructions, correlation dimension $\left(D_{2}\right)$ and Kolmogorov entropy $\left(K_{2}\right)$ calculations based on Kantz's algorithm [19] were applied to analyze the glottal area and acoustical time series.

\section{A. Phase space reconstructed}

The reconstructed phase space shows that the dynamic behavior of a signal can be reconstructed by the time delay[18]. A time series with length $N$ is measured and recorded as $x\left(t_{1}\right), x\left(t_{2}\right), \ldots, x\left(t_{N}\right)$, where $x\left(t_{i}\right) \in \mathrm{R}, t_{i}=t_{0}+i \tau(i=1,2, \ldots, N)$ at the discrete time interval $\tau$. Then, the time delay vector $\tau$ creates the reconstructed phase space as:

$$
X(t)=\{x(t), x(t-\tau), \ldots, x(t-(m-1) \tau)\}
$$

where $m$ is the embedding dimension.

\section{B. Correlation dimension $\mathrm{D}_{2}$}

The correlation dimension $\left(\mathrm{D}_{2}\right)$ is a quantitative measure that specifies the number of degrees of freedom needed to describe a dynamic system. The correlation dimension can be calculated as follows:

$$
D_{2}=\lim _{r \rightarrow 0} \lim _{N \rightarrow \infty} \frac{\ln C(N, r)}{\ln r},
$$

Where $r$ is the radius around $X_{i}$, and the correlation integral $C(N, r)$ is

$$
C(N, r)=\frac{1}{N(N-1)} \sum_{i=1}^{N} \sum_{\substack{j=1 \\ i \neq j}}^{N} \theta\left(r-\left\|X_{i}-X_{j}\right\|\right),
$$

Where the Heaviside function $\theta(x)$ satisfies

$$
\theta(x)= \begin{cases}1, & x>0 \\ 0, & x \leq 0\end{cases}
$$

Using the correlation dimension method, chaos has been found to distinguish different dynamic characteristics from white noise. The estimated $D_{2}$ of white noise does not converge with the increase of embedding dimension $m$, while the estimated $D_{2}$ of a chaotic system converges to a finite value with the 
increase of $m$. Therefore, a more complex system has a higher dimension, meaning that more degrees of freedom may be needed to describe its dynamic state $[4,5,19]$.

\section{Kolmogorov entropy $K_{2}$}

Kolmogorov entropy $\left(K_{2}\right)$ is a description of the rate of information loss in a dynamic system $[4,5,19] . \mathrm{K}_{2}$ is a useful measure in distinguishing chaos from stochastic noise. For a regular system with little movement, $K_{2}=0$; for a stochastic system, $K_{2}$ approaches infinity; and for a chaotic system, $K_{2}$ is a positive constant. That is, the larger Kolmogorov entropy is, the more complex the system is.

Kolmogorov entropy is usually estimated by second Renyi entropy $K_{2}$. The relationship between $K_{2}$ and $C(N, r)$ is as follows:

$$
K_{2}=\lim _{m \rightarrow \infty} \lim _{r \rightarrow 0} \lim _{N \rightarrow \infty} \frac{1}{m \tau} \ln \frac{C(m, r)}{C(m+1, r)}
$$

When discrete time interval $\tau$ and embedding dimension $m$ are fixed values,

\subsection{Statistical analysis}

Correlation dimension and Kolmogorov entropy of the glottal areas and acoustical signals from each voice signal type were analyzed using SigmaStat 3.5(Systat Software, San Jose, CA). The voice signal groups were compared using individual Mann-Whitney rank sum tests. A significance level of $p<0.05$ was used throughout.

\section{Results}

The extracted glottal area time series and recorded acoustic time series of 4 signal types have been illustrated in Fig.2. The vibratory patterns have a strong correlation with the corresponding acoustical waveforms for type 1,2 and 3 signals, but not for that of type 4 . The type 1 pattern displays regular and periodic waveforms, as shown in Fig. 2(a). In Fig. 2(b), the type 2 pattern appears periodic, alternating between small and large amplitudes that are defined as a subharmonic wave [1]. The type 3 and type 4 
waveforms have no apparent periodicity, as shown in Fig. 2(c) and (d), but the type 4 signals are characterized by a high frequency signal.

Fig. 3 (a)-(d) displays the corresponding frequency spectrum of the vibratory patterns and acoustical signals for each voice signal type. The frequency spectrums of type 1 vibratory patterns and acoustical signals show a discrete fundamental frequency and integer multiples harmonic frequencies $n f_{0}$, $n=1,2 \ldots N$. Type 2 signals exhibit subharmonic frequencies $f_{0} / 2$. Type 3 signals display a broadband frequency spectrum and frequency peaks that mainly exist at low frequencies. In contrast to the type 3, type 4 signals exhibit a smearing of energy across a broad frequency range with no apparent energy peaks, as is characteristic of broadband white noise.

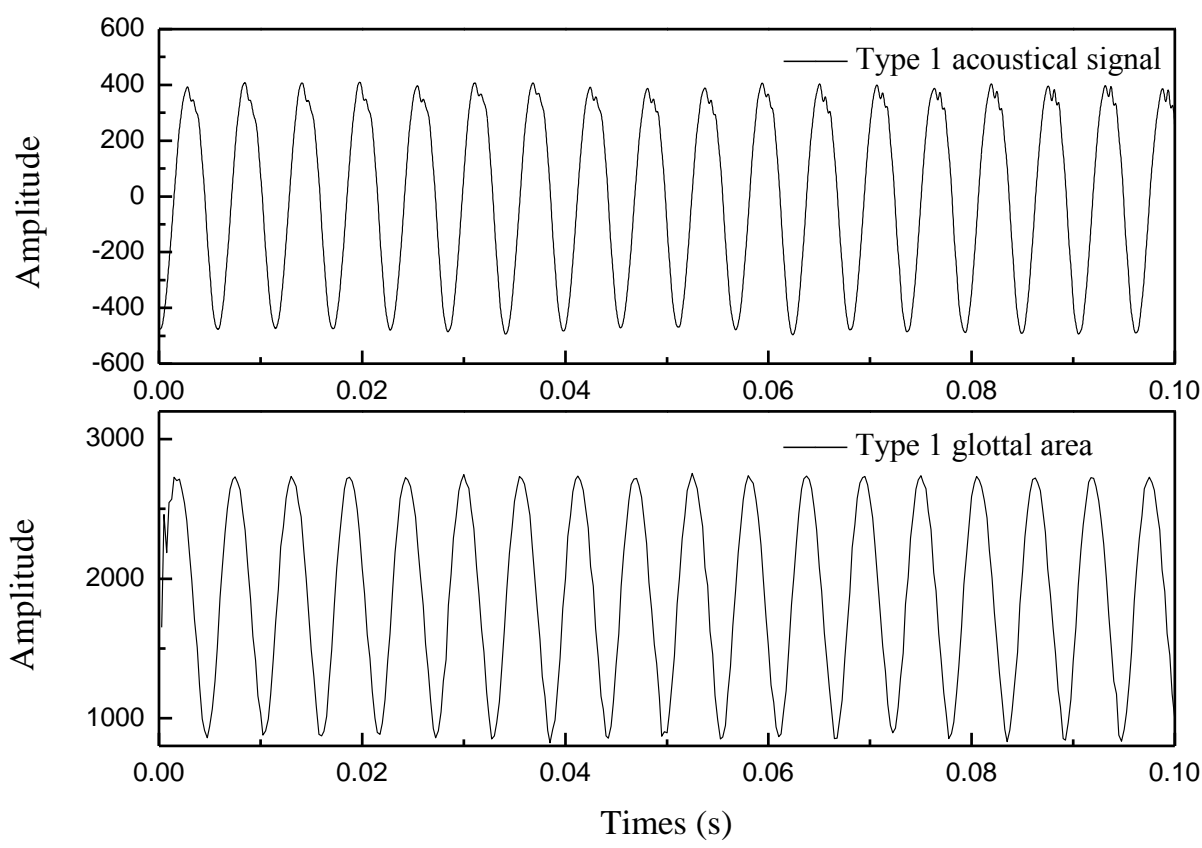

(a) 


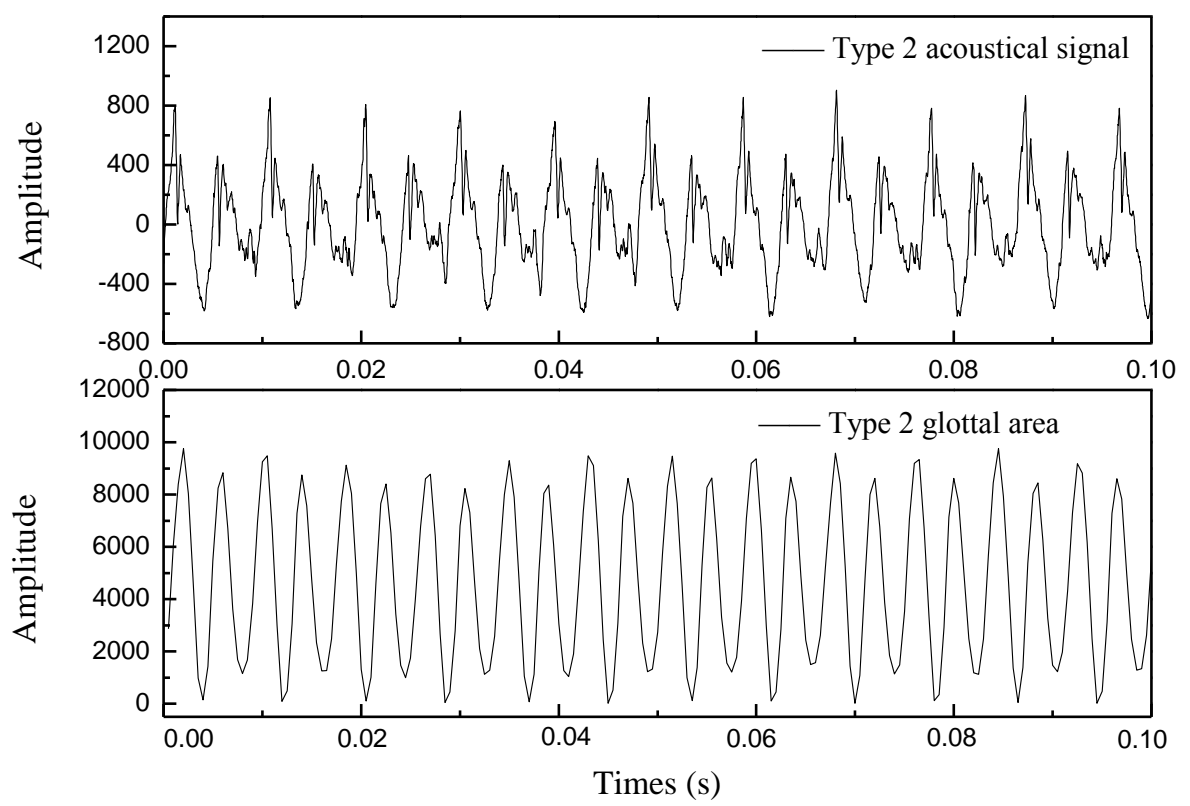

(b)
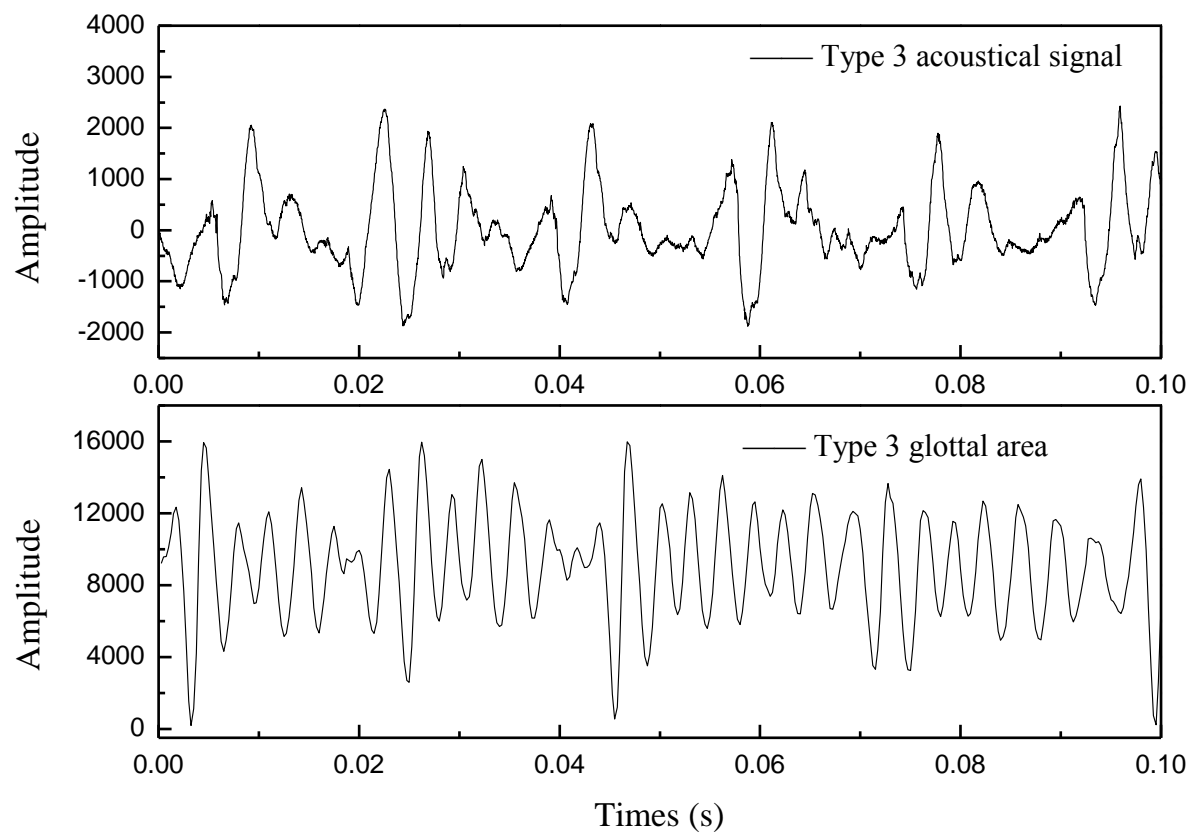

(c) 

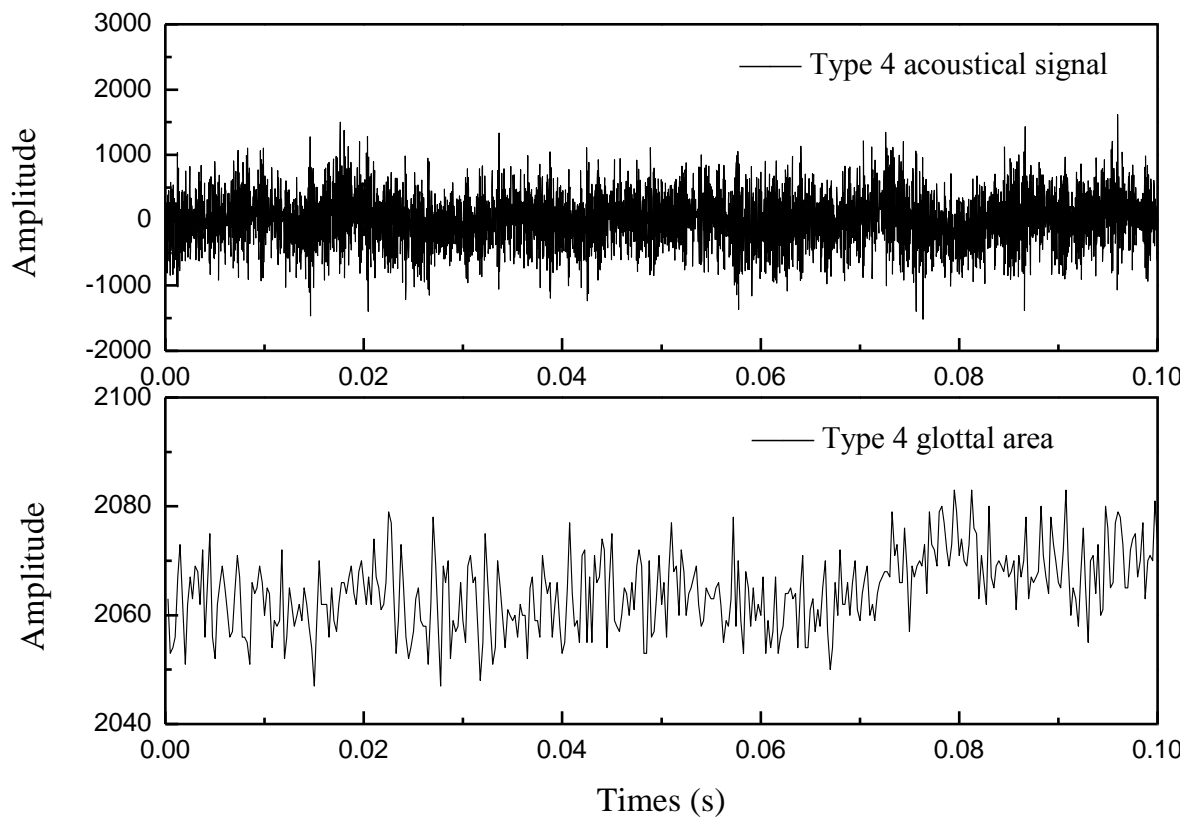

(d)

Fig. 2 The time series of acoustical signal and glottal area of

(a) Type 1, (b) Type 2, (c) Type 3 and (d) Type 4.

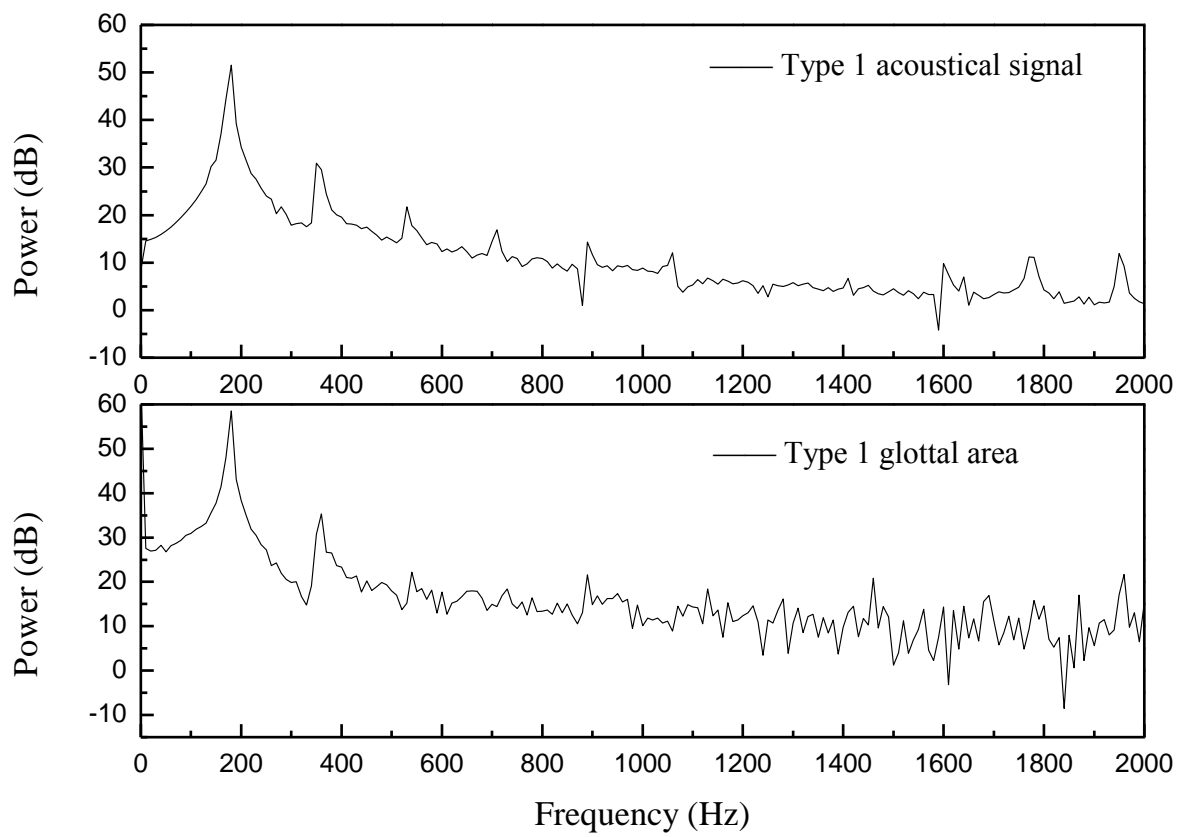

(a) 


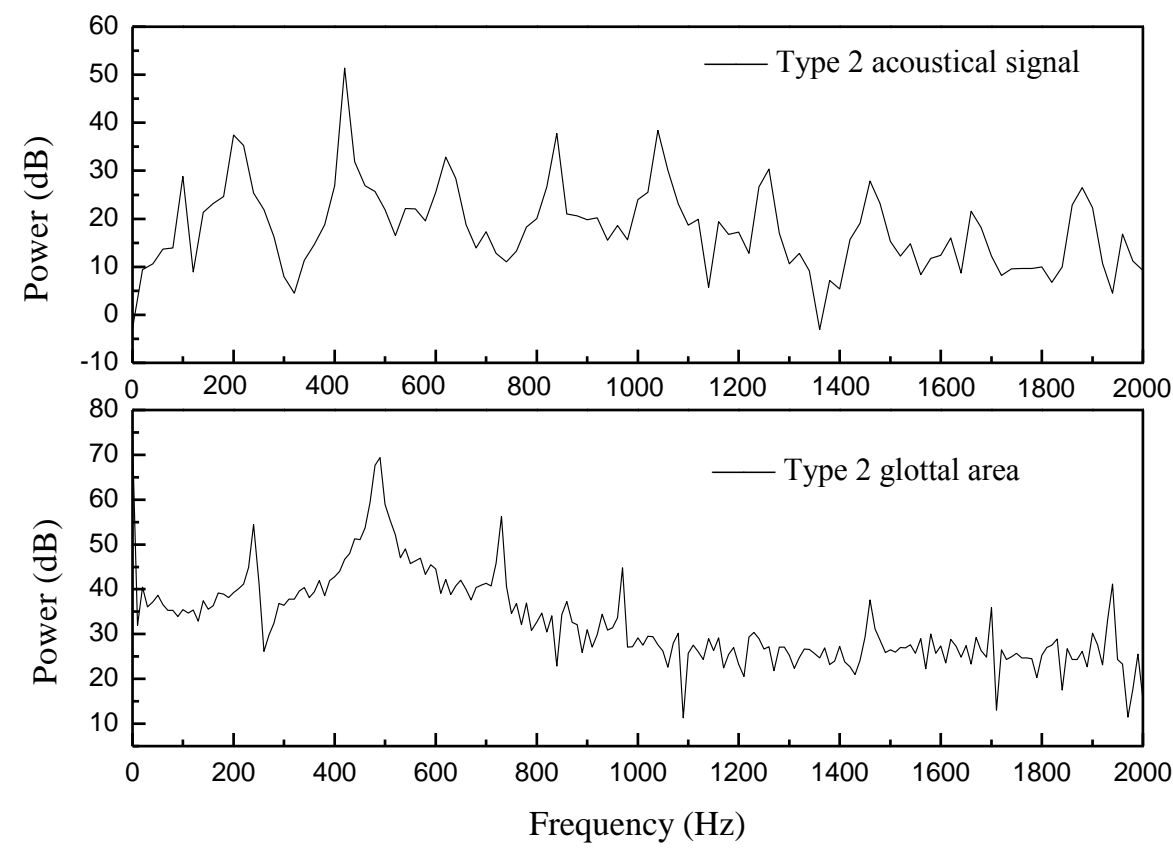

(b)

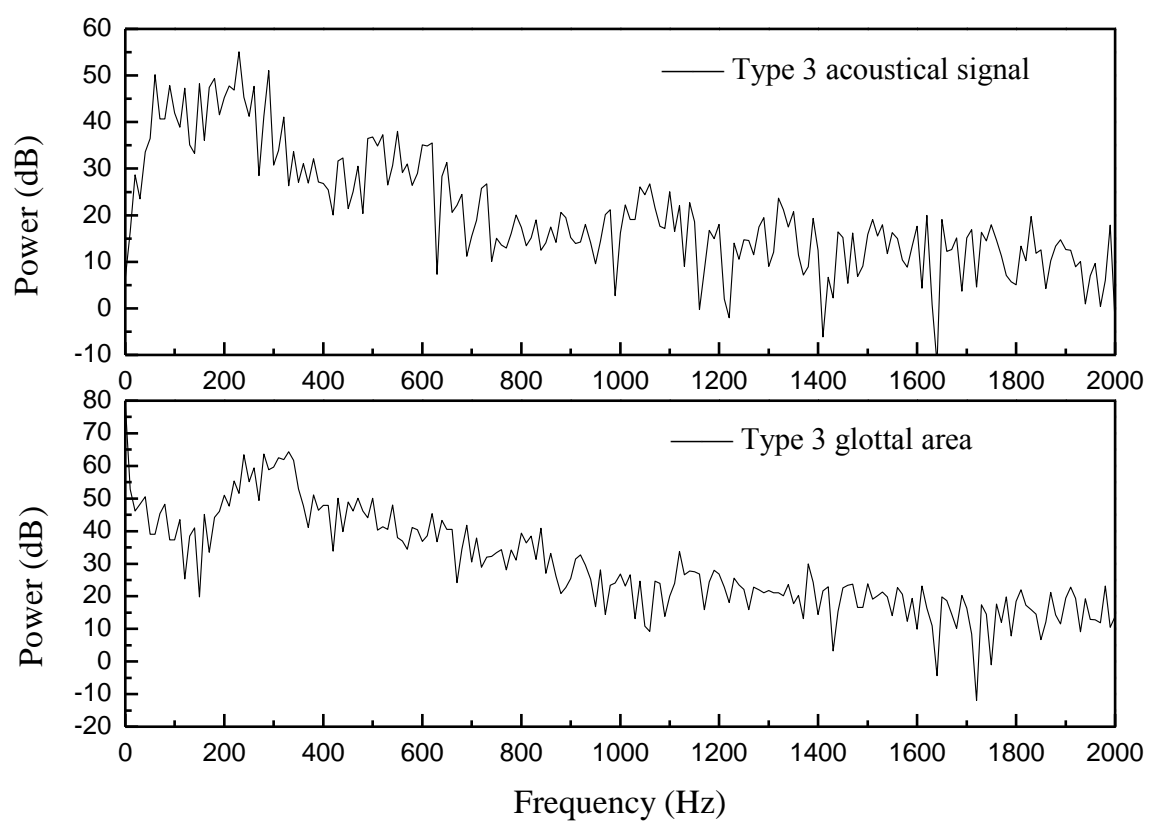

(c) 


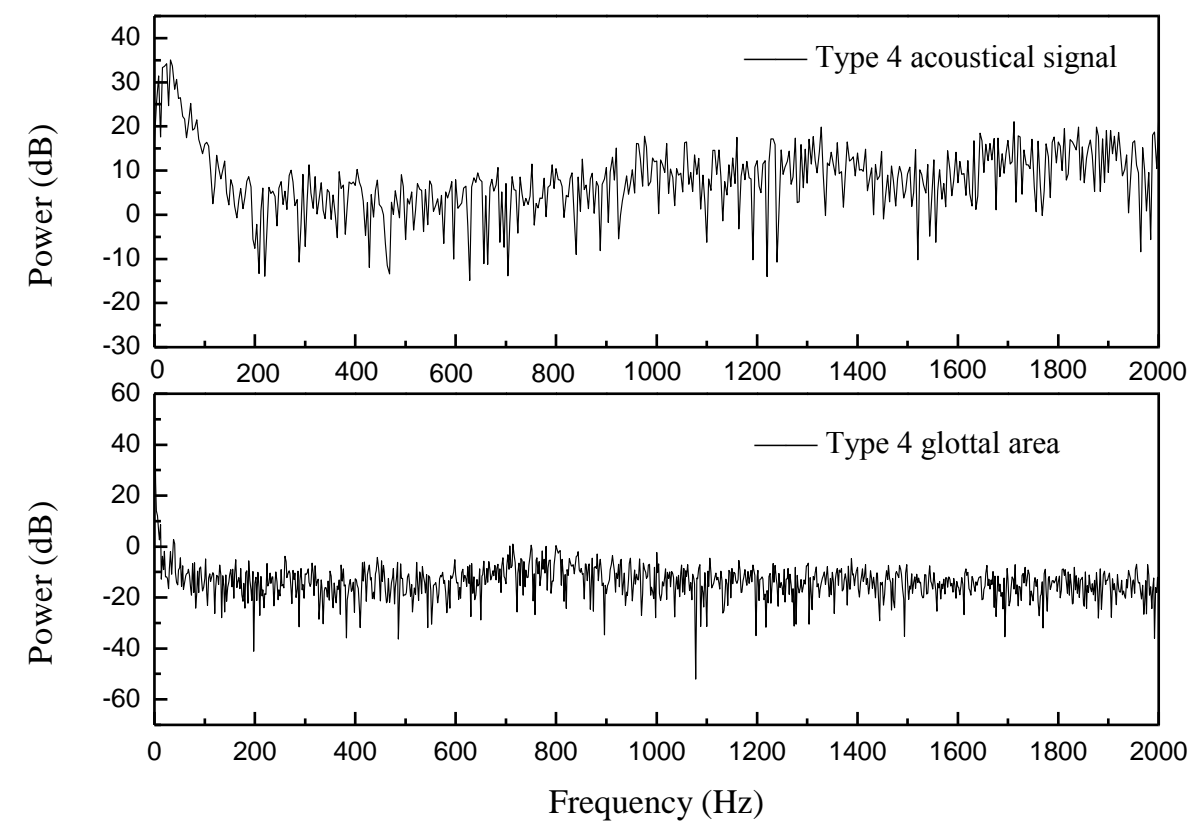

(d)

Fig. 3 The frequency spectrum of acoustical signal and glottal area of

(a) Type 1, (b) Type 2, (c) Type 3 and (d) Type 4.

Both glottal area and acousitical time series of all four voice signal types were analyzed by nonlinear dynamic analysis methods. According to the Kantz algorithm, the relationship between correlation integral $\log C(r)$ and $\ln (r)$ of the 4 types of acoustical signals and glottal areas were calculated by increasing the embedding dimension $m=1,2, \ldots, 12$, as seen in Fig. 4.

The results were illustrated in Fig. 5. According to Fig.5 (a), the $D_{2}$ values of type 1, type 2 and type 3 acoustical signals converged to $1.05 \pm 0.001,2.18 \pm 0.003$, and $4.2 \pm 0.001$ respectively. The complexity of the voice signal was directly correlated with correlation dimension. However, the $D_{2}$ estimate of type 4's acoustical signal was infinite with the increasing of embedding dimension $m$, similar to white noise. As shown in Fig. 5(b), the $D_{2}$ values of type 1, type 2 and type 3 glottal areas converged to $1.08 \pm 0.001,2.27 \pm 0.001$ and $3.19 \pm 0.002$ similar to the acoustical signals. Interestingly, the $D_{2}$ estimate of the type 4 glottal areas was finite and converged to $0.11 \pm 0.004$.

In order to reflect the difference of the correlation dimensions between the vibratory pattern and acoustical signal of 4 types signals, the statistical results were given in Fig. 6. The correlation dimensions of type 1, type 2, type 3 glottal area and acoustical signal series showed strong correlations 
( $p=0.341, p=0.974, p=0.431)$. However, the correlation dimension of type 4 acoustical signals showed no convergence with increasing embedding dimension. In order to quantify the difference of the type 4 signal from type $1,2,3$, we chose the values at embedding dimension $m=12$ as the correlation dimension of type 4 acoustical signals, as seen in Fig.6. This demonstrates that the statistical data of type 4 voice signals has obvious discrepancies between the vibratory and acoustical system $(p<0.001)$.

Additionally, the statistical graph of Kolmogorov entropy provided strong evidence of the inconsistency between the vibratory and acoustical systems of type 4 voice signals. As illustrated in Fig. 7, the $K_{2}$ of the glottal area and acoustical signal series of type 1,2, and 3 voice signals showed remarkable consistency $(p=0.430, p=0.743, p=0.533)$. Whereas in type 4 , the $K_{2}$ of the glottal area series was close to 0 , but that of the acoustical signal series was infinite. Similar to the method for choosing $D_{2}$ of the type 4 signals, we chose the values at an embedding dimension $m=12$ as the Kolmogorov entropy. This resulted in significant differences between the vibratory and acoustical systems $(p<0.001)$
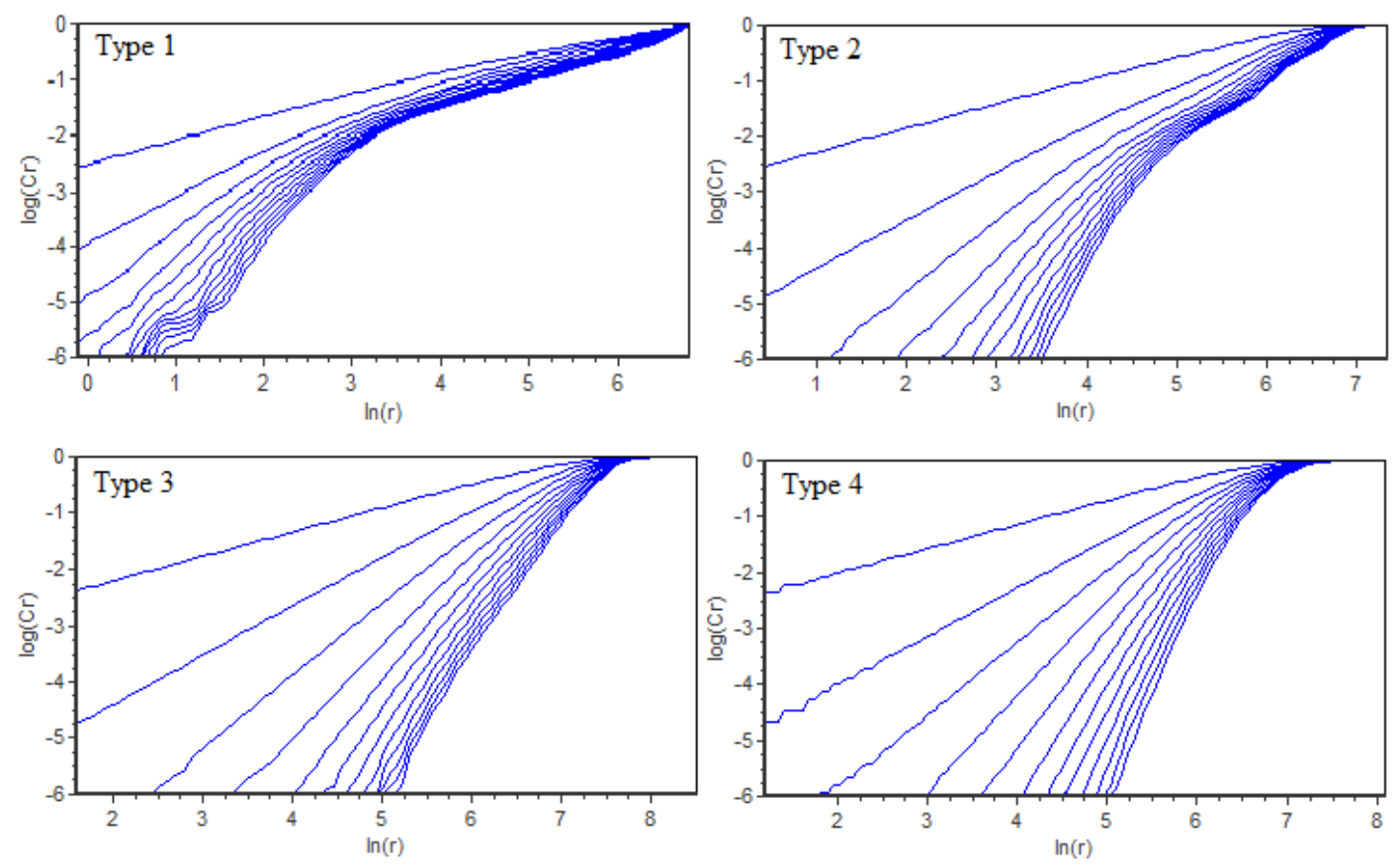

(a) 

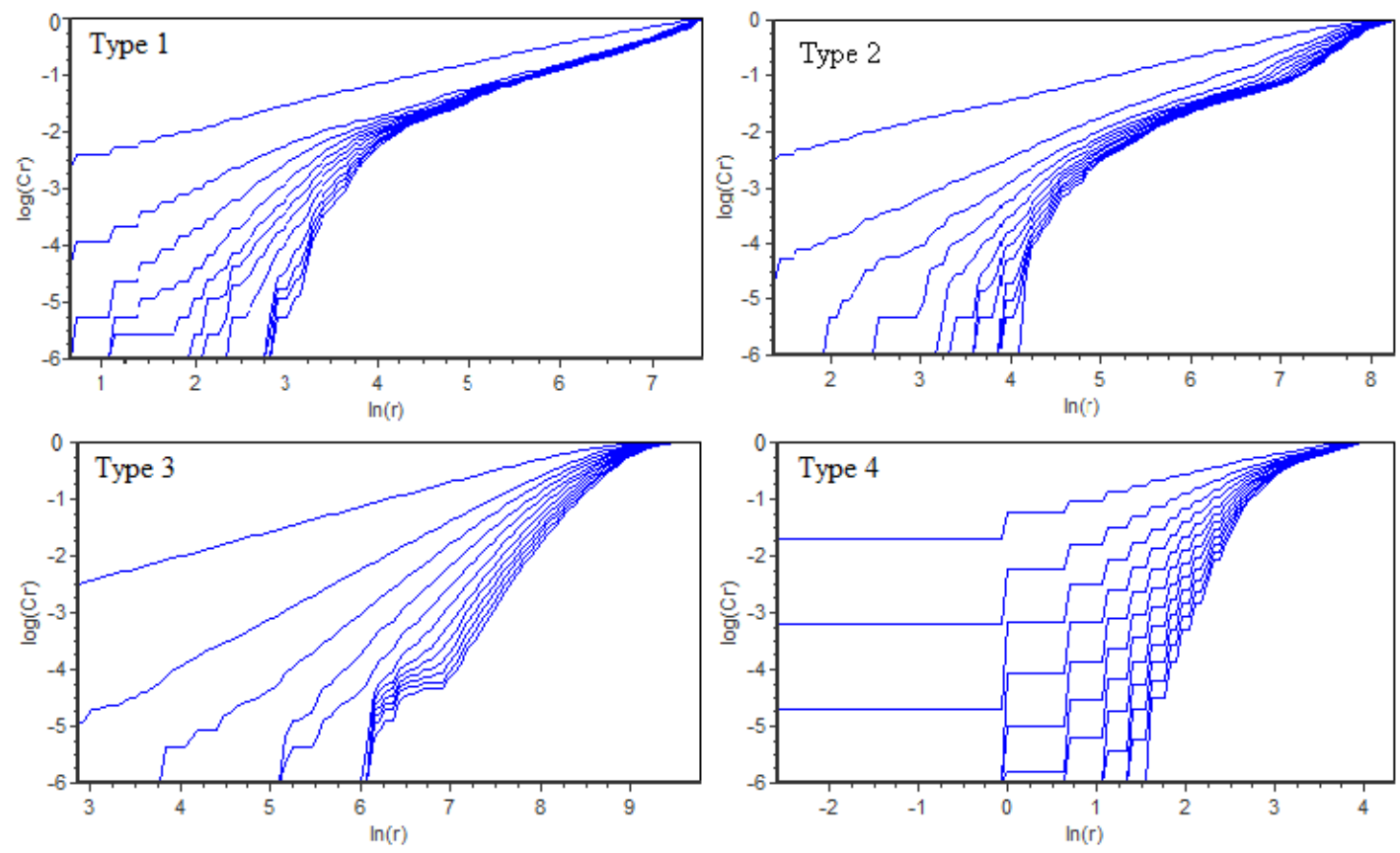

(b)

Fig. 4 The correlation integral $\log C(\mathrm{r})$ vs $\ln (\mathrm{r})$ of the 4 types (a) acoustical signals and (b) glottal areas.

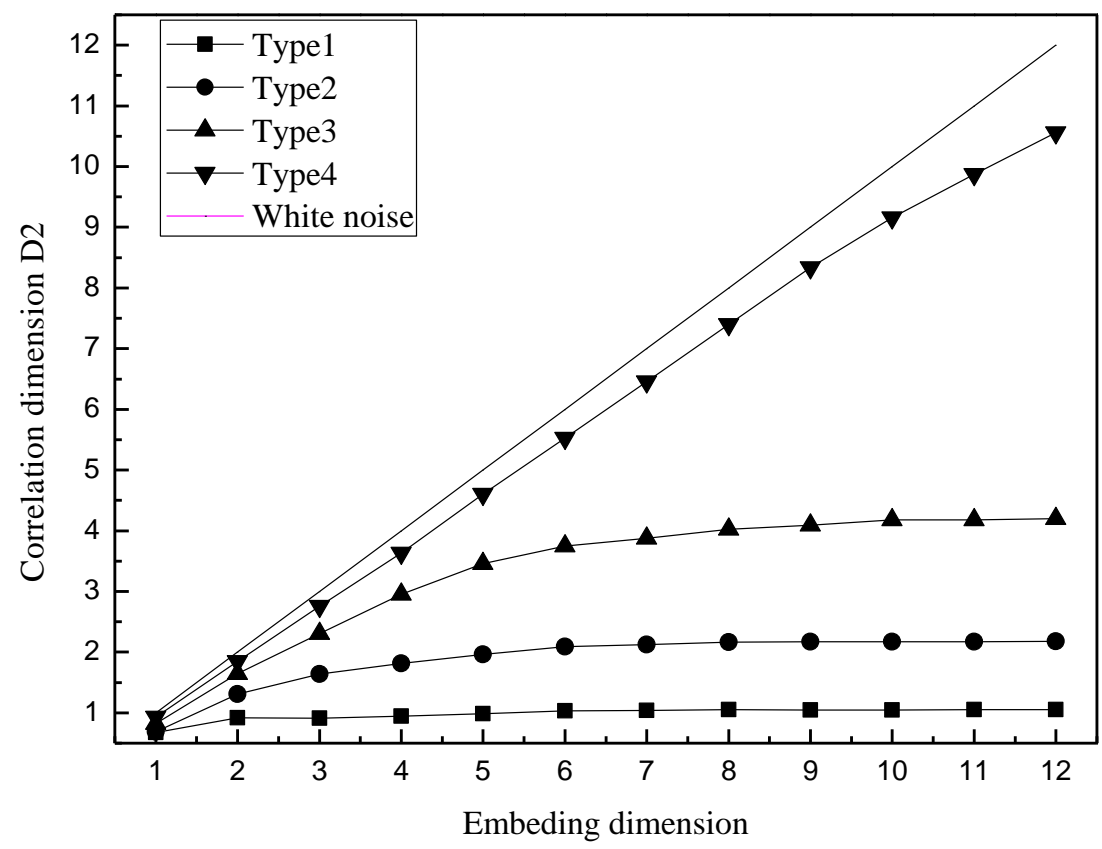

(a) 


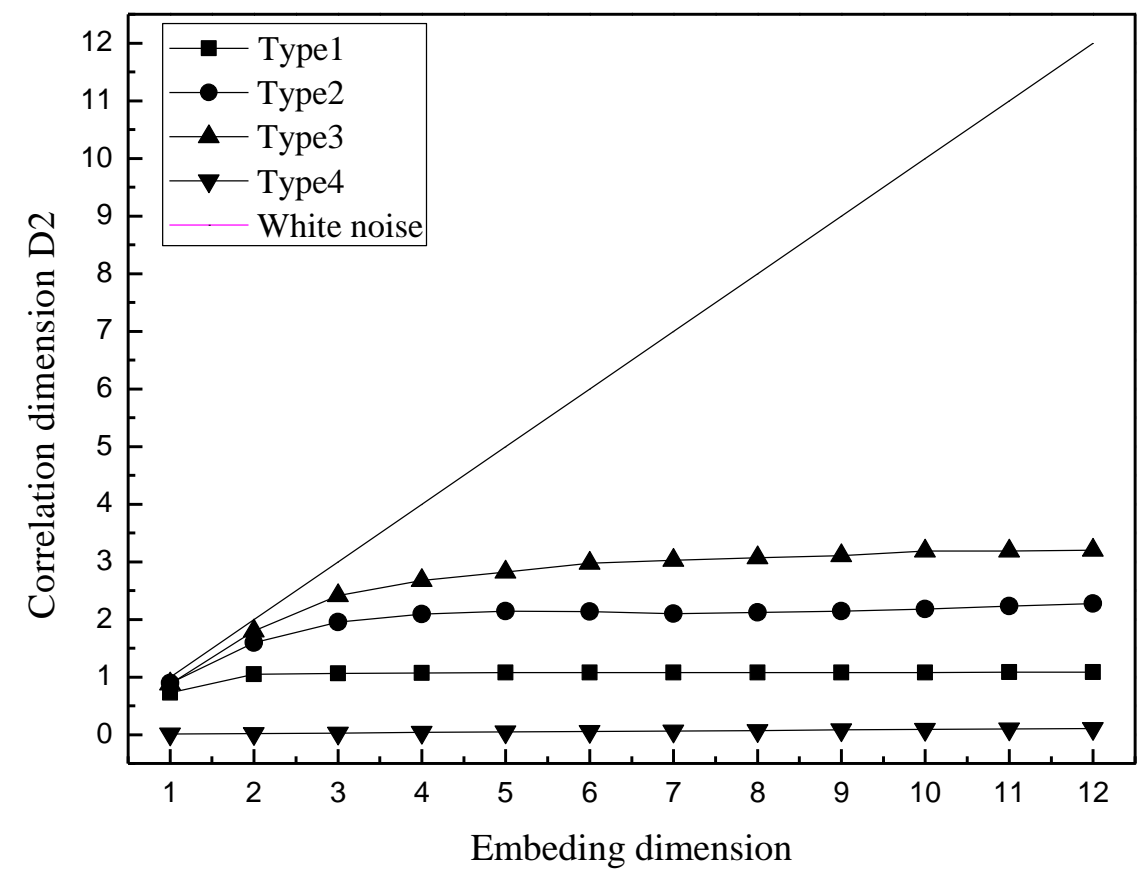

(b)

Fig. 5 (a) The estimated dimension versus embedding dimension $m$ of the type 1,2, 3 and 4 acoustical signals

(b)The estimated dimension versus embedding dimension $m$ of the type 1, 2, 3 and 4 glottal areas 


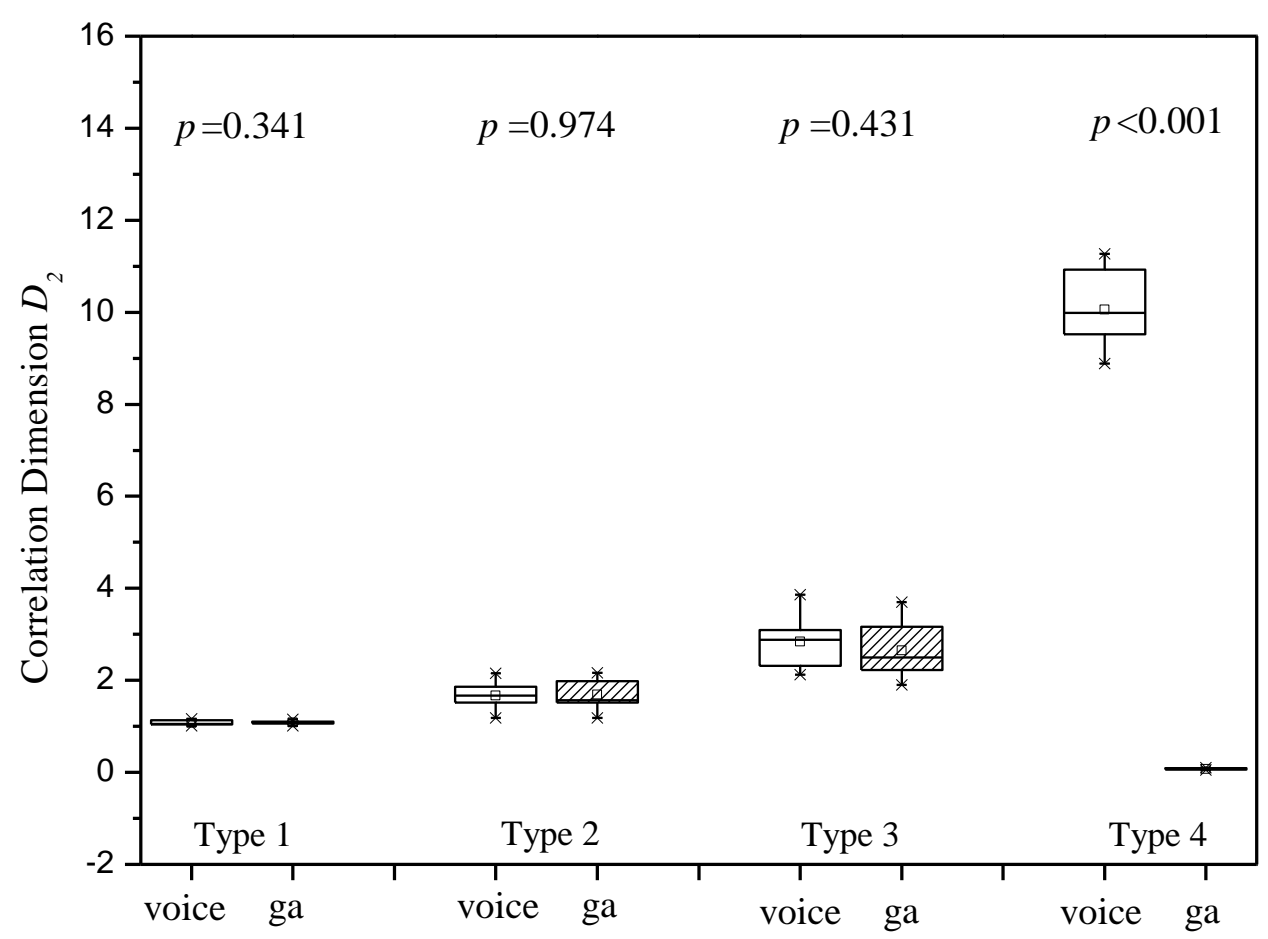


Fig. 6 The correlation of correlation dimension D2 between acoustical signals (voice) and glottal areas (ga) of 4 types.

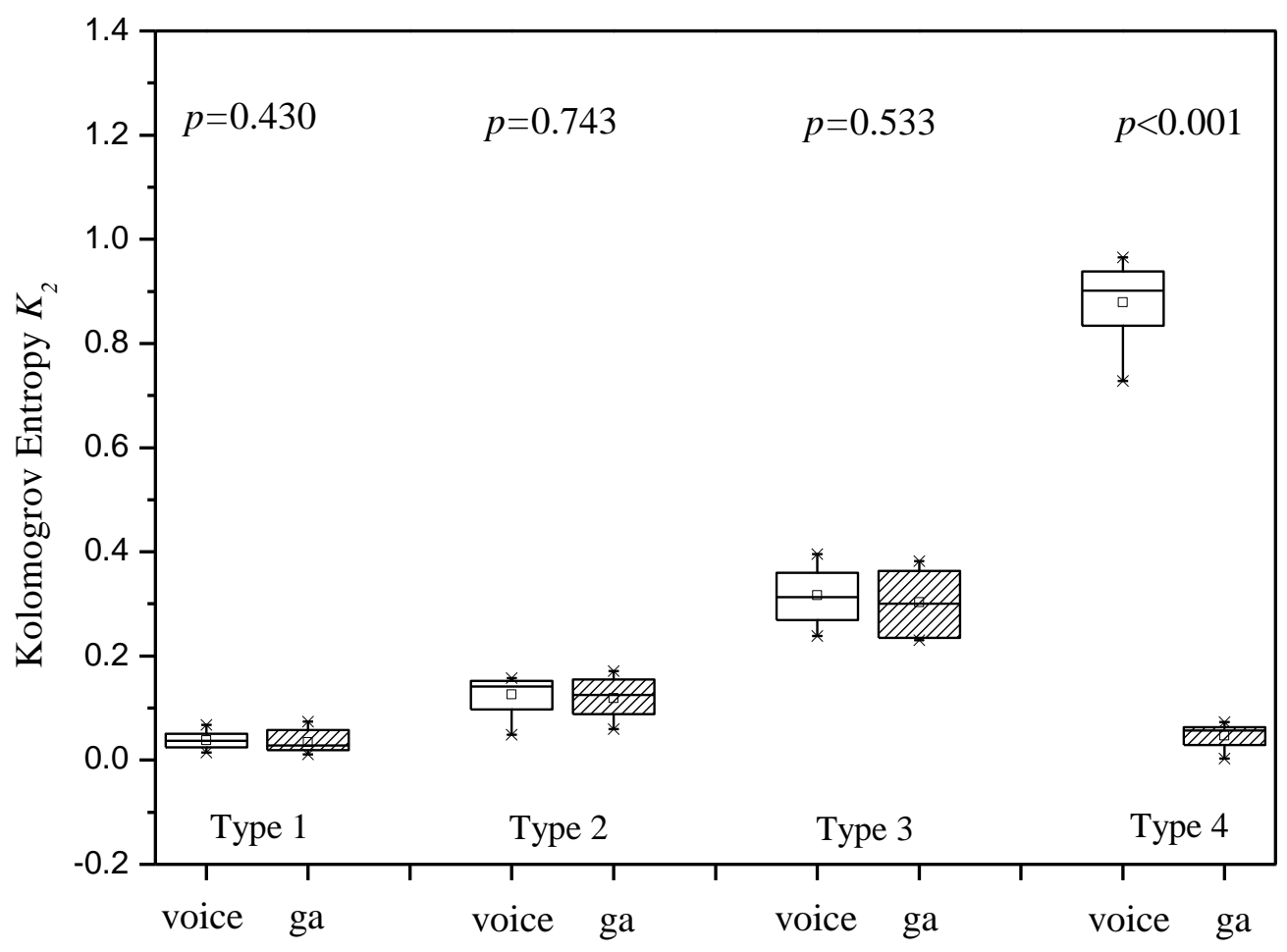

Fig.7 The correlation of Kolmogorov entropy $K_{2}$ between acoustical signals (voice) and glottal areas (ga) of 4 types.

\section{Discussion}

In this study, we collected the vibratory images and acoustical signals of the four voice signal types from excised larynges. This study aimed at investigating the differences between the vibratory patterns and modulated acoustical systems of these signal types. We developed Sprecher's classification scheme of acoustic voice signals because it was not previously able to provide information about the vibratory dynamics of the vocal folds due to complicating factors such as turbulent noise and vocal tract filtering. High-speed images reflect the original vibratory patterns of the vocal folds, especially for type 3 and type 4 voice signals. Current acoustical analysis methods such as perturbation analysis and nonlinear dynamic analysis are used to analyze voice signals. However, traditional perturbation techniques are not applicable to type 3 and type 4 signals because their outputs are neither valid nor reliable for aperiodic voice signals $[1-6,20]$. Thus, nonlinear dynamic analysis techniques are needed to provide valuable information about the nature of these complex systems [1-6]. In this paper, vibratory images were 
recorded using high-speed imaging techniques and analyzed using glottal area time series, frequency spectra, and nonlinear dynamics. The acoustical signals were analyzed using the same methods.

Fig. 2 and Fig. 3 demonstrate that vibratory patterns have a strong correlation with the corresponding acoustical waveforms for type 1,2 and 3 voice signals, but not for that of type 4. As shown in Fig. 2(a) and 3(a), type 1 displayed periodic time series and discrete frequency spectra, consistent with the findings of previous studies $[2,16]$. This finding confirms that type 1 vibrations are dominated by first order harmonic components. The estimated correlation dimension of the glottal areas and acoustical signals of type 1 signals were both close to 1, as seen in Fig.5. This suggests that type 1 voice signals are a one-dimensional system. In contrast to type 1, subharmonic components and half of fundamental frequency $\left(f_{0} / 2\right)$ existed in the glottal areas, acoustical signal time series, and the corresponding frequency spectrum of type 2 signals, as seen in Fig. 2(b) and 3(b). These results suggest that type 2 signals consist of several modes that make the correlation dimension of type 2 higher than that of type 1, as shown in Fig. 5.

Both type 3 and type 4 signals were aperiodic without any clear pattern in either amplitude or frequency, as seen in Fig. 2(c), 2(d), 3(c) and 3(d). The frequency spectra of type 3 voice signals was captured by the energy peaks, while type 4 signals resembled that of white noise. This is seen in Fig. 3(c) and 3(d). The estimated $D_{2}$ of type 3 acoustical signals was finite, while that of type 4 was characterized by infinite dimensionality, as illustrated by Fig. 5(a). Surprisingly, the estimated dimension of the type 4 glottal area time series was finite, conflicting with the results of the type 4 acoustical signals. Additionally, the entropy of the type 4 glottal area time series was close to 0 , while the acoustic signals $\mathrm{K}_{2}$ was much higher, as shown in Fig. 6 and Fig.7. This is because type 4 acoustical signals are disordered voice signals with a large breathy component produced by turbulence in the vocal tract. This breathiness destroys the self-similarity property of the chaotic system and dominates the signal as high-frequency noise [2]. However, the glottal areas extracted from high-speed images of type 4 can eliminate the effects of turbulence and vocal tract filtering, which inverts the original movement patterns of the vocal fold without high-frequency noise. Thus, it is reasonable that the estimated dimensions $\left(D_{2}\right)$ and Kolmogorov entropy $\left(K_{2}\right)$ of type 4 glottal area time series are finite and converge to 0 , as seen in this study.

Fig.7 demonstrates that the $K_{2}$ values of the type 4 glottal area time series are similar to that of the type 1 voice signal. This is because the $K_{2}$ is theoretically equal to 0 for regular movements. Therefore, 
it is suggested that correlation dimensions $\left(D_{2}\right)$ better represents the differences among the four signal types than Kolmogorov entropy $\left(K_{2}\right)$ and could be an effective tool in distinguishing the four signal types.

Current methods of voice signal analysis are time consuming and not ideal for quantifying the complexity of disordered voice characteristics. The results of this study indicate that high speed vibratory imaging combined with nonlinear analysis such as correlation dimension $\left(D_{2}\right)$ could provide an objective metric capable of evaluating all four voice signal types. This approach could allow for the expansion of evidence-based practices in dysphonia management as well as explore the mechanism underlying type 4 voice production. Future research can utilize the approach followed in this paper to determine the critical turbulent energy, or the amount of turbulent energy required to produce type 4 voice signals. A more complete understanding of the mechanism responsible for producing type 4 voice would provide a better approach to the treatment of these disorders as well as examine which pathologies are usually responsible for producing that signal. Lastly, this new analysis method can track how a voice signal changes during a treatment period, allowing the clinician to monitor the efficacy of a specific intervention.

\section{Conclusion}

Four types of vocal fold vibrations were directly observed via high-speed images of excised canine larynges. Next, the glottal area time series were extracted from these images. Nonlinear dynamic analysis methods were used to quantify the four types of vocal fold glottal area time series and acoustical signals. The results proved that the nonlinear dynamic parameters of correlation dimension $\left(D_{2}\right)$ and Kolmogorov entropy $\left(K_{2}\right)$ effectively distinguish all four voice signal types based on their acoustical signals. The calculations of the vibration patterns and acoustical signals for type 1,2 , and 3 voice signals showed strong correlations. However, type 4 signals did not show this correlation. While the nonlinear dynamic calculations of the vibration patterns of type 4 signals were approximately 0 , their acoustic signal calculations were infinite. This phenomena is due to the large breathy component that is seen in type 4 acoustical signals, which is produced by turbulence in the vocal tract. Therefore, 
we conclude that high-speed images provide a more original representation of the type 4 vocal fold vibratory pattern and a more effective method to explore the mechanism behind type 4 voice signals.

\section{Acknowledgments}

This work was financially supported in part by the National Science Foundation of China (Grants No. 41276040 and No. 11174240) and the Natural Science Foundation of Fujian Province of China (Grant No. 2012J06010). The project was sponsored by the Scientific Research Foundation for the Returned Overseas Chinese Scholars, State Education Ministry.

\section{References}

[1] Titze IR. Workshop on acoustic voice analysis: summary statement. Denver, CO; National Center for Voice and Speech; 1995.

[2] Sprecher A, Olszewski A, Jiang JJ, Zhang Y. Updating signal typing in voice: addition of type 4 signals. J Acoust Soc Am 2010, 127(6):3710-3716

[3] Zhang Y, Jiang JJ, Wallace SM, Zhou L. Comparison of nonlinear dynamic methods and perturbation methods for voice analysis. J Acoust Soc Am 2005, 118:2551-2560.

[4] Zhang Y, McGilligan C, Zhou L, Vig M, Jiang JJ. Nonlinear dynamic analysis of voice before and after surgical excision of vocal polyps. J Acoust Soc Am 2004, 115: 2270-2277.

[5] Jiang JJ, Zhang Y, Ford CN. Nonlinear dynamics of phonations in excised larynx experiments. J Acoust Soc Am 2003, 114: 2198-2205.

[6] Zhang Y, Jiang JJ. Nonlinear dynamic analysis in signal typing of pathological human voices. Electron Lett 2003,39:1021-1023.

[7] Karnell MP, Chang A, Smith A, Hoffman H. Impact of signal type on validity of voice perturbation measures. NCVS Status and Progress Report 1997,11: 91-94.

[8] Behrman A, Agresti CJ, Blumstein E, Lee N. Microphone and electroglottographic data from dysphonic patients: type1, 2 and 3 signals. J Voice 1998,12: 249-260.

[9] Witt RE, Taylor LN, Regner MF, BS, Jiang JJ, Effects of surface dehydration on mucosal wave amplitude and frequency in excised canine larnges, Otolaryngol Head Neck Surgery 2011; 144:108-113. [10] Jiang JJ, Zhang Y, Kelly MP, Bieging ET, Hoffman MR, An automatic method to quantify mucosal 
waves via videokymography, The Laryngoscope 2008; 118:1504-1510.

[11] Jiang JJ, Chang CIB, Raviv JR, Gupta S, Banzali FM, Jr, Hanson DG, Quantitative study of mucosal wave via video-kymography in canine larynes, The Laryngoscope 2000; 110:1567-1573.

[12] Yan Y, Chen X, Bless D, Automatic tracing of vocal fold motion from high-speed digital images, Journal of Biomedical Engineering 2006; 53:1394-1400.

[13] Tao C, Zhang Y, Jiang JJ, Extracting physiologically relevant parameters of vocal folds from high-speed video image series, Journal of Biomedical Engineering 2007; 54:794-801.

[14] Svec JG, Schutte HK, Videokymography: high-speed line scanning of vocal fold vibration, Journal of Voice 1996; 10:201 - 205.

[15] Svec JG, Schutte HK, Sram F, Videokymography: A new high-speed method for the examination of vocal-fold vibrations, Otorinolaryngol a Foniatrie 1999; 48:155 - 162.

[16] Zhang Y, Krausert CR, Kelly MP, Jiang JJ, Typing vocal fold vibratory patterns in excised larynx experiments via digital kymography, Ann Otol Rhinol Laryngol 2009; 118:598-605.

[17] Zhang Y, Bieging E, Tsui H, Jiang J J. Efficient and effective extraction of vocal fold vibratory patterns from high-speed digital imaging. Journal of Voice, 2010, 24:21-29.

[18] Packard NH, Crutchfield JP, Farmer JD, and Shaw RS. Geometry from a time series. Phys. Rev. Lett. $45,712,1980$.

[19] Holger Kantz and Thomas Schreiber. Nonlinear time series analysis. Cambridge : the United Kingdom at the University Press, 1997.

[20] Karnell MP, Chang A, Smith A and Hoffman H.'Impact of signal type of validity of voice perturbation measures ”, NCVS Status Prog. Rep. 11,91-94,1997. 